\title{
Dose-volume histogram parameters and patient-reported EPIC- Bowel domain in prostate cancer proton therapy
}

\author{
Gabriella F. Bulman ${ }^{1}$, Ronik S. Bhangoo ${ }^{1}$, Todd A. DeWees ${ }^{1,2}$, Molly M. Petersen ${ }^{2}$, Cameron S. Thorpe ${ }^{1}$, \\ William W. Wong ${ }^{1}$, Jean Claude M. Rwigema ${ }^{1}$, Thomas B. Daniels ${ }^{1}$, Sameer R. Keole ${ }^{1}$, Steven E. Schild ${ }^{1}$, \\ Carlos E. Vargas ${ }^{1}$ \\ ${ }^{1}$ Department of Radiation Oncology, Mayo Clinic, Phoenix, AZ, USA \\ ${ }^{2}$ Department of Health Sciences Research, Mayo Clinic, Scottsdale, AZ, USA
}

Received: March 31, 2021

Revised: May 11, 2021

Accepted: May 14, 2021

Correspondence:

Gabriella F. Bulman

Department of Radiation Oncology,

Mayo Clinic, 5777 E Mayo Blvd,

Phoenix, AZ 85054, USA

Tel: +1-480-515-6296

E-mail: bulman.gabriella@mayo.edu ORCID:

https://orcid.org/0000-0003-4781-1196
Purpose: To analyze rectal dose and changes in quality of life (0OL) measured with the Expanded Prostate and Cancer Index Composite (EPIC) bowel domain in patients being treated for prostate cancer with curative-intent proton beam therapy (PBT) within a large single-institution prospective registry.

Materials and Methods: Data was collected from 243 patients with localized prostate cancer treated with PBT from 2016 to 2018. The EPIC survey was administered at baseline, end-of-treatment, 3, 6 , and 12 months, then annually. Dose-volume histogram (DVH) parameters for the rectum were computed, and rectal dose was analyzed using $\operatorname{BED}(\alpha / \beta=3), \mathrm{EQD}_{2 \mathrm{~Gy}}$ and total dose. Repeated measures mixed models were implemented to determine the effect of patient, clinical, and treatment factors (including DVH) on patient-reported bowel symptom burden (EPIC-Bowel).

Results: Treatment overall resulted in changes in EPIC-Bowel scores (baseline score $=93.7$ ), most notably at end-of-treatment (90.6) and 12 months (89.7). However, they returned to baseline at 36 months (92.9). On multivariate modeling, rectal BED $D_{25}$ (Gy) $\geq 23 \%$ was significantly associated with decline in $\mathrm{QOL}$ scores measuring bother ( $\mathrm{p}<0.01 ; 4.06$ points different).

Conclusion: Rectal doses, specifically BED $D_{25}(G y) \geq 23 \%$, are significantly associated with decline in bowel bother-related $\mathrm{QOL}$ in patients undergoing definitive radiotherapy for localized prostate cancer. This study demonstrates BED as an independent predictor of bowel $\mathrm{QOL}$ across dose fractionations of PBT.

Keywords: Prostatic neoplasms, Radiation therapy, Quality of life

\section{Introduction}

Prostate cancer is the most common cancer in American men, with roughly 200,000 new cases and 30,000 deaths annually [1]. Quality of life (QOL) issues are important for prostate cancer patients who generally survive for years after therapy [2-5]. External beam irradiation is one of the treatment options for localized prostate cancer. Proton beam therapy (PBT) is a unique form of radiotherapy (RT) that delivers high-dose, targeted particles to tumors while minimizing radiation dose to surrounding healthy tissue [6,7]. Previous research has generally included clinician evaluated toxicities resulting from PBT [8-11]. However, the relationship between dose volume parameters and quality of life using the Expanded Prostate Cancer Index Composite (EPIC) scores remains unclear.

The EPIC survey measures health-related quality of life across a variety of disease-specific domains such as urinary, bowel, and sexual function [12]. Furthermore, the survey has expanded on previous instruments like the University of California Los Angeles Pros- 
tate Cancer Index (UCLA-PCI) to capture irritative and hormone therapy-related side effects [13]. Thus, the survey provides a comprehensive and valuable measure of $\mathrm{QOL}$ as patients self-report their symptoms throughout the course of treatment.

Previous studies have demonstrated statistically and clinically significant changes in EPIC-Bowel scores for patients receiving PBT for prostate cancer $[14,15]$. We sought to investigate this further with analysis of the survey subdomains across different fraction schemes, using conventional fractionation, hypofractionation and extreme hypofractionation. We present a prospective analysis on how PBT affects QOL in patients undergoing curative treatment for prostate cancer.

\section{Materials and Methods}

\section{IRB statement}

The Institutional Review Board of Mayo Clinic Arizona approved the study and all patients provided written informed consent (No. 19007866). This trial was registered with ClinicalTrials.gov (NCT012 55748).

\section{Eligibility criteria}

Adult males undergoing definitive radiotherapy for localized prostate cancer from January 1, 2016 to December 31, 2018 were originally assessed for eligibility. To be included, patients must have received proton RT with curative intent, and completed at least one pre-RT and one post-RT EPIC questionnaire. Patients with node-positive or metastatic disease were excluded. The final number of patients enrolled was 243 .

\section{Radiation therapy}

Patients underwent computed tomography (CT) simulation with pelvic immobilization, a full bladder, and a rectal balloon. Carbon fiducial markers were placed prior to simulation. Some patients also had a rectal spacer placed between the prostate and rectum at the discretion of the treating physician. A treatment planning magnetic resonance imaging (MRI) was obtained and co-registered with CT images for target delineation. In patients who could not undergo MRI scans, treatments were planned using $\mathrm{CT}_{\text {, positron }}$ emission tomography (PET), and bone scans. The clinical target volume (CTV) encompassed the entire prostate gland and proximal seminal vesicles. In cases of T3b disease, the entire seminal vesicles were included in CTV. An optimization target volume (OTV) was created from the CTV using a margin of 2-3 mm posteriorly and 3 $\mathrm{mm}$ elsewhere. Treatment was delivered with right and left lateral beams. The constructed OTV included an additional $5 \mathrm{~mm}$ in the beam direction distally and proximally due to range uncertainty. Usual proton beams were oriented laterally left and right. Image guidance with matching to carbon fiducial markers was performed to confirm daily set up.

For the purpose of this study, conventional fractionation denotes dose per fraction between 1.8 and 2 Gy (relative biological effectiveness [RBE]). Hypofractionation was defined as treatment regimens of more than 5 fractions with over 2 Gy (RBE) per fraction. Extreme hypofractionation (stereotactic body radiation therapy [SBRT]) was defined as treatments of 5 fractions or less, typically $\geq 6$ Gy (RBE) per fraction. Spot scanning proton beam was used for all treatments.

\section{Dosimetric data}

Rectal dose-volume histogram (DVH) parameters collected from treatment plans were computed with $D_{x}$ signifying dose (Gy) delivered to a percentage of rectal tissue volume. $V_{x}$ denoted the percentage of rectum volume receiving at least a given dose (Gy). Assuming an alpha-beta ratio of 3 , both the biologically effective dose (BED $=D \times\left(1+\frac{d}{\alpha / \beta}\right)$ and equivalent dose in 2 Gy fractions $\left(\mathrm{EQD}_{2 \mathrm{~Gy}}=D \times \frac{d+\frac{\alpha}{\beta}}{2 G y+\frac{\alpha}{\beta}}\right)$ of each rectal DVH parameter was calculated [16]. We assumed the rectum to have an alpha-beta ratio of 3 for BED and $\mathrm{EQD}_{2 \mathrm{~Gy}}$ calculations.

Institutional guidelines for conventional fractionation, hypofractionation, and extreme fractionation were utilized when determining rectal dose constraints. For conventional fractionation: $V_{65}(G y)$ $\leq 50 \%$; hypofractionation: $V_{61}(G y) \leq 15 \%$; and extreme hypofractionation: $V_{33.5}(G y) \leq 15 \%$. For the hypofractionation schemes, the higher dose constraints were approximately $10 \%$ greater than conventional fractionation protocols: BED of 98.5 Gy (65 Gy nominal) for conventional, 110 Gy (61 Gy nominal) for hypofractionation, and $108 \mathrm{~Gy}$ (33.5 Gy nominal) for extreme hypofractionation.

\section{Toxicity assessments}

Toxicity was measured with the Common Terminology Criteria for Adverse Events (version 5.0).

\section{6. $\mathrm{QOL}$ measures}

The EPIC is a survey tool used to measure health-related $\mathrm{OOL}$ through assessment of urinary, bowel, sexual, and hormonal burden during treatment of prostate cancer [12]. Patients completed the EPIC-50 and AUASI-17 (American Urological Association Symptom Index) at the following intervals: baseline, end of treatment, and 3, $6,12,24$, and 36 months. A higher EPIC score implies less bowel symptom burden. Previous analyses have established the minimally 
important difference (MID) as 4-6 points within the EPIC-Bowel domain $[17,18]$.

\section{Statistical analysis}

Rectal dose was analyzed using BED (alpha/beta = 3), EQD ${ }_{2 G y}$ and total dose. Univariate analysis utilizing repeated measures mixed models was utilized to determine the "best" DVH parameter associated with differences in patient-reported QOL. An outcome-oriented approach (with alpha-adjustment to control for family-wise error) was used to determine the optimal dichotomization of the DVH parameter of interest with the largest association to changes in patient-reported $\mathrm{QOL}$. Multivariate repeated measures mixed models with unstructured covariance matrices were implemented to determine the effect of patient, clinical, and treatment factors (including DVH) on patient-reported bowel symptom burden (EPIC-Bowel overall, function, and bother scores).

\section{Results}

\section{Patient and tumor characteristics}

The present analysis includes 243 patients from January 1, 2016 to December 31, 2018, treated for prostate cancer in accordance with institutional protocols. Patient and tumor characteristics are displayed in Table 1. The median pre-RT PSA (prostate-specific antigen) value was $6.1 \mathrm{ng} / \mathrm{mL}$ (range, 1.3 to $129 \mathrm{ng} / \mathrm{mL}$ ), median T stage was T1c (range, T1b-T3b), median Gleason score was 7 (range, 6 to 10), and median age was 71 years (range, 52 to 91 years).

\section{Radiotherapy characteristics and outcomes}

The median follow-up time was 20 months (range, 2.5 to 40 months). Dose fractionations are presented in Table 2. Conventional fractionation was used in 117 patients (48\%), with a median dose of 1.8 Gy and range of 1.8-2.0 Gy. Moderate hypofractionation was used in 84 patients (34\%), with a median dose of $2.5 \mathrm{~Gy}$ and a range of 2.5-3.0 Gy. Extreme hypofractionation was used in 42 patients (17.3\%), with all patients receiving 7.6 Gy. Median rectal $\mathrm{BED}_{\text {mean }}$ was $12.45 \mathrm{~Gy}$ (range, 1.74 to $50.5 \mathrm{~Gy}$ ) and median rectal $\mathrm{EQD}_{2 \text { mean }}$ was $7.47 \mathrm{~Gy}$ (range, 1.04 to $30.32 \mathrm{~Gy}$ ). Median post-RT PSA at first follow-up was $0.22 \mathrm{ng} / \mathrm{mL}$ (range, 0 to $29.71 \mathrm{ng} / \mathrm{mL}$ ). One patient (0.4\%) died by the last follow-up due illness unrelated to cancer and radiation treatment.

\section{DVH analyses}

DVH parameters were determined by $B E D, E_{26 D^{\prime}}$ and total dose. Each continuous parameter was tested for associations with significant decline in EPIC scores at intervals of 5\% in volume and 5 Gy
Table 1. Patient and tumor characteristics $(n=243)$

\begin{tabular}{|c|c|}
\hline Characteristic & Value \\
\hline Age (yr) & 71 (52-91) \\
\hline \multicolumn{2}{|l|}{ Race } \\
\hline White & $227(93.4)$ \\
\hline Other & $16(6.6)$ \\
\hline \multicolumn{2}{|l|}{ ECOG performance status } \\
\hline 0 & $198(81.5)$ \\
\hline 1 & $44(18.1)$ \\
\hline 2 & $1(0.4)$ \\
\hline 1 or 2 & $45(18.5)$ \\
\hline Pre-RT PSA (ng/ml) & $6.140(0.3-1289)$ \\
\hline$<10$ & $185(76.1)$ \\
\hline $10-20$ & $44(18.1)$ \\
\hline$>20$ & $14(5.8)$ \\
\hline \multicolumn{2}{|l|}{ T stage } \\
\hline $\mathrm{T} 1-\mathrm{T} 2 \mathrm{a}$ & $188(77.4)$ \\
\hline $\mathrm{T} 2 \mathrm{~b}-\mathrm{T} 2 \mathrm{c}$ & $32(13.2)$ \\
\hline T3-T4 & $22(9.1)$ \\
\hline \multicolumn{2}{|l|}{ Gleason score, total } \\
\hline 6 (group 1) & $35(14.4)$ \\
\hline 7 (group 2 or 3 ) & $146(60)$ \\
\hline $3+4$ (group 2) & 91 (37.4) \\
\hline 4+3 (group 3) & $55(22.6)$ \\
\hline 8 (group 4) & $36(14.8)$ \\
\hline 9-10 (group 5) & $26(10.7)$ \\
\hline Biopsy cores taken & $13(2-34)$ \\
\hline Biopsy cores positive & $5(1-25)$ \\
\hline \multicolumn{2}{|l|}{ MRI } \\
\hline Yes & $230(94.7)$ \\
\hline No & 13 (5.3) \\
\hline \multicolumn{2}{|l|}{ MRI results } \\
\hline Extraprostatic extension & $27(11.1)$ \\
\hline Seminal vesicle invasion & $5(2.1)$ \\
\hline \multicolumn{2}{|l|}{ Androgen deprivation therapy } \\
\hline Yes & $155(63.8)$ \\
\hline No & 88 (36.2) \\
\hline
\end{tabular}

Values are presented as median (range) or number (\%).

ECOG, Eastern Cooperative Oncology Group; RT, radiotherapy; PSA, prostate-specific antigen; MRI, magnetic resonance imaging.

for dose. The univariately significant DVH parameters are displayed in Table 3. For table brevity, large ranges of non-significant values are listed at intervals of $10 \mathrm{~Gy}$ instead of $5 \mathrm{~Gy}$. Across BED and $E_{2 G y}$ significant parameters included $D_{20}, D_{25}, D_{30}, D_{95}$, and $D_{100}$. For total dose, $\mathrm{D}_{25}, \mathrm{D}_{30}, \mathrm{D}_{35}, \mathrm{D}_{95}, \mathrm{D}_{100}, \mathrm{~V}_{65}$, and $\mathrm{V}_{70}$ were significant. Based on statistical significance and clinical interpretability, BED $D_{25}(G y)$ was selected as the "best" continuous predictor for differences in EPIC-Bowel scores over time. 
Table 2. Dose fractionation with $\mathrm{BED}$ and $\mathrm{EQD}_{2 \mathrm{~Gy}}$ transformations

\begin{tabular}{|c|c|c|c|c|c|}
\hline & \multicolumn{2}{|c|}{ Prostate } & \multicolumn{2}{|c|}{ Rectum } & \multirow{2}{*}{ Total number (\%) } \\
\hline & $\operatorname{BED}(\alpha / \beta=1.5)$ & $\mathrm{EQD}_{2 \mathrm{~Gy}}$ & $\operatorname{BED}(\alpha / \beta=3)$ & $\mathrm{EQD}_{2 \mathrm{~Gy}}$ & \\
\hline \multicolumn{6}{|c|}{ Dose fractionation (SBRT) } \\
\hline 38 Gy/5 fx & 230.5 & 98.8 & 134.3 & 80.6 & $42(17.3)$ \\
\hline \multicolumn{6}{|l|}{ Hypofractionation } \\
\hline $60 \mathrm{~Gy} / 20 \mathrm{fx}$ & 180 & 77.1 & 120 & 72 & $9(3.7)$ \\
\hline $67.5 \mathrm{~Gy} / 25 \mathrm{fx}$ & 189 & 81 & 128.3 & 77.0 & $26(10.7)$ \\
\hline 70 Gy/28 fx & 180 & 77.1 & 128.3 & 77 & $49(20.2)$ \\
\hline \multicolumn{6}{|c|}{ Conventional fractionation } \\
\hline $75.6 \mathrm{~Gy} / 42 \mathrm{fx}$ & 166.3 & 71.3 & 121.0 & 72.6 & $1(0.4)$ \\
\hline $77.4 \mathrm{~Gy} / 43 \mathrm{fx}$ & 170.3 & 73 & 123.8 & 74.3 & $8(3.3)$ \\
\hline 78 Gy/39 fx & 182 & 78 & 130 & 78 & $2(0.8)$ \\
\hline $79.2 \mathrm{~Gy} / 44 \mathrm{fx}$ & 174.2 & 74.7 & 126.7 & 76.0 & $106(43.6)$ \\
\hline
\end{tabular}

$\mathrm{BED}$, biologically effective dose; $\mathrm{EQD}_{2 \mathrm{~Gy}}$ equivalent dose in 2 Gy fractions; SBRT, stereotactic body radiation therapy.

Table 3. Univariate analysis of DVH parameters used in selecting BED $D_{25} G y(p=0.027)$ as the optimal predictor for differences in EPIC-Bowel, to be evaluated through subsequent multivariate analysis

\begin{tabular}{|c|c|c|c|c|c|c|}
\hline & \multicolumn{2}{|c|}{ BED } & \multicolumn{2}{|c|}{$\mathrm{EQD}_{2 \mathrm{~Gy}}$} & \multicolumn{2}{|c|}{ Total dose } \\
\hline & Median & p-value & Median & $p$-value & Median & $p$-value \\
\hline Dmean & 12.050 & NS & 7.230 & NS & 8.080 & NS \\
\hline $\mathrm{D}_{0}$ & 132.090 & NS & 79.250 & NS & 72.070 & 0.0008 \\
\hline$D_{5}$ & 87.040 & NS & 52.220 & NS & 53.030 & NS \\
\hline$D_{10}$ & 43.410 & NS & 26.050 & NS & 31.560 & NS \\
\hline$D_{15}$ & 20.090 & NS & 12.050 & NS & 16.510 & NS \\
\hline$D_{20}$ & 9.770 & 0.0338 & 5.860 & 0.0338 & 8.790 & NS \\
\hline$D_{25}$ & 4.990 & 0.0270 & 2.990 & 0.0270 & 4.630 & 0.0402 \\
\hline$D_{30}$ & 2.790 & 0.0349 & 1.680 & 0.0349 & 2.690 & 0.0352 \\
\hline$D_{35}$ & 1.730 & NS & 1.040 & NS & 1.700 & 0.0490 \\
\hline$D_{40}$ & 1.250 & NS & 0.750 & NS & 1.230 & NS \\
\hline$D_{50}$ & 0.720 & NS & 0.430 & NS & 0.710 & NS \\
\hline$D_{60}$ & 0.400 & NS & 0.240 & NS & 0.400 & NS \\
\hline$D_{70}$ & 0.202 & NS & 0.121 & NS & 0.200 & NS \\
\hline$D_{80}$ & 0.070 & NS & 0.040 & NS & 0.070 & NS \\
\hline$D_{90}$ & 0.007 & NS & 0.004 & NS & 0.007 & NS \\
\hline $\mathrm{D}_{95}$ & 0.001 & 0.0013 & 0.001 & 0.0013 & 0.001 & 0.0013 \\
\hline$D_{100}$ & 0 & 0.0004 & 0 & 0.0004 & 0 & 0.0004 \\
\hline$V_{10}$ & 19.800 & NS & 16.410 & NS & 19.030 & NS \\
\hline$V_{20}$ & 15.040 & NS & 11.930 & NS & 13.470 & NS \\
\hline$V_{30}$ & 12.480 & NS & 8.910 & NS & 10.420 & NS \\
\hline$V_{40}$ & 10.410 & NS & 7.210 & NS & 7.810 & NS \\
\hline$V_{50}$ & 8.910 & NS & 5.380 & NS & 5.720 & NS \\
\hline$V_{60}$ & 7.810 & NS & 3.920 & NS & 3.360 & NS \\
\hline$V_{65}$ & 7.400 & NS & 3.040 & NS & 2.390 & 0.0566 \\
\hline$V_{70}$ & 6.770 & NS & 2.070 & NS & 0.700 & 0.0093 \\
\hline$V_{80}$ & 5.740 & NS & 0 & NS & - & - \\
\hline$V_{90}$ & 4.740 & NS & - & - & - & - \\
\hline$V_{100}$ & 3.920 & NS & - & - & - & - \\
\hline$V_{110}$ & 2.790 & NS & - & - & - & - \\
\hline$V_{120}$ & 1.640 & NS & - & - & - & - \\
\hline$V_{125}$ & 1.010 & NS & - & - & - & - \\
\hline
\end{tabular}

DVH, dose-volume histogram; BED, biologically effective dose; $\mathrm{EQD}_{2 \mathrm{~Gy}}$ equivalent dose in 2 Gy fractions; EPIC, Expanded Prostate Cancer Index Composite; NS, not significant. 


\section{EPIC scores}

Globally, treatment had expected effects over EPIC-Bowel scores which returned to baseline over time, with a median baseline score (93.7), end-of-treatment (90.6), 3 months (92.1), 6 months (92.7), 12 months (89.7), 24 months (93.3), and 36 months (92.9). EPIC score decline from baseline was significant at end-of-treatment ( $p$ $=0.04)$ and 12 months post-treatment $(p<0.01)$. However, within 24 months, patient QOL demonstrated a return to pre-RT levels $(p=0.99)$.

Extreme hypofractionation was associated with a 4.58 point decrease in total EPIC-Bowel scores ( $p<0.01$ ) when compared to conventional fractionation, while hypofractionation was not associated with a significant decline $(-1.42$ points, $p=0.07)$. Both extreme hypofractionation $(-4.06$ points, $p<0.01)$ and hypofractionation $(-2.47$ points, $p<0.01)$ were significantly associated with decline in bowel function compared to conventional fractionation. Extreme hypofractionation was significantly associated with a decrease in bother scores $(-5.12$ points, $p<0.01)$, while hypofractionation was not $(-0.40$ points, $p=0.66)$.

Rectal dose affected $\mathrm{OOL}$ with the strongest DVH signals noted for BED $D_{25}$ (Gy) with an optimal cut point of $\geq 23 \%$. This parameter was significant after controlling for age, body mass index (BMI), race, Eastern Cooperative Oncology Group (ECOG) performance status $\geq 1$, Gleason score, clinical T stage, androgen deprivation therapy (ADT) use, and fractionation schedule. For overall EPIC-Bowel scores, the dose of BED $D_{25}$ (Gy) $\geq 23 \%$ was associated with an average decrease of 2.73 points compared to those $<23 \%$ $(p<0.01)$. The effects of radiotherapy on bowel were subdivided into the domains of function and bother. A dose of BED $D_{25}$ (Gy) $\geq 23 \%$ did not significantly affect bowel function ( -1.44 points, $p$ $=0.13)$. However, reported bother was significantly worse $(p<$ 0.01) with a score difference of 4.06 points (Fig. 1).

\section{Discussion and Conclusion}

In a single, large prospective registry ( $\mathrm{n}=243)$, we determined BED to be an independent predictor for bowel-related QOL. Overall, EPIC-Bowel scores were significantly affected by BED $D_{25}$ (Gy) $\geq 23 \%$ ( $p<0.01$ ). On multivariate modeling, this parameter was significantly associated with a decline in QOL scores measuring bother ( $p<0.01,4.06$ points different) as opposed to function.

The MID for the EPIC-Bowel domain has been established as 4-6 points $[17,18]$. In other words, this is the threshold for which patients can detect clinically meaningful changes in their symptom

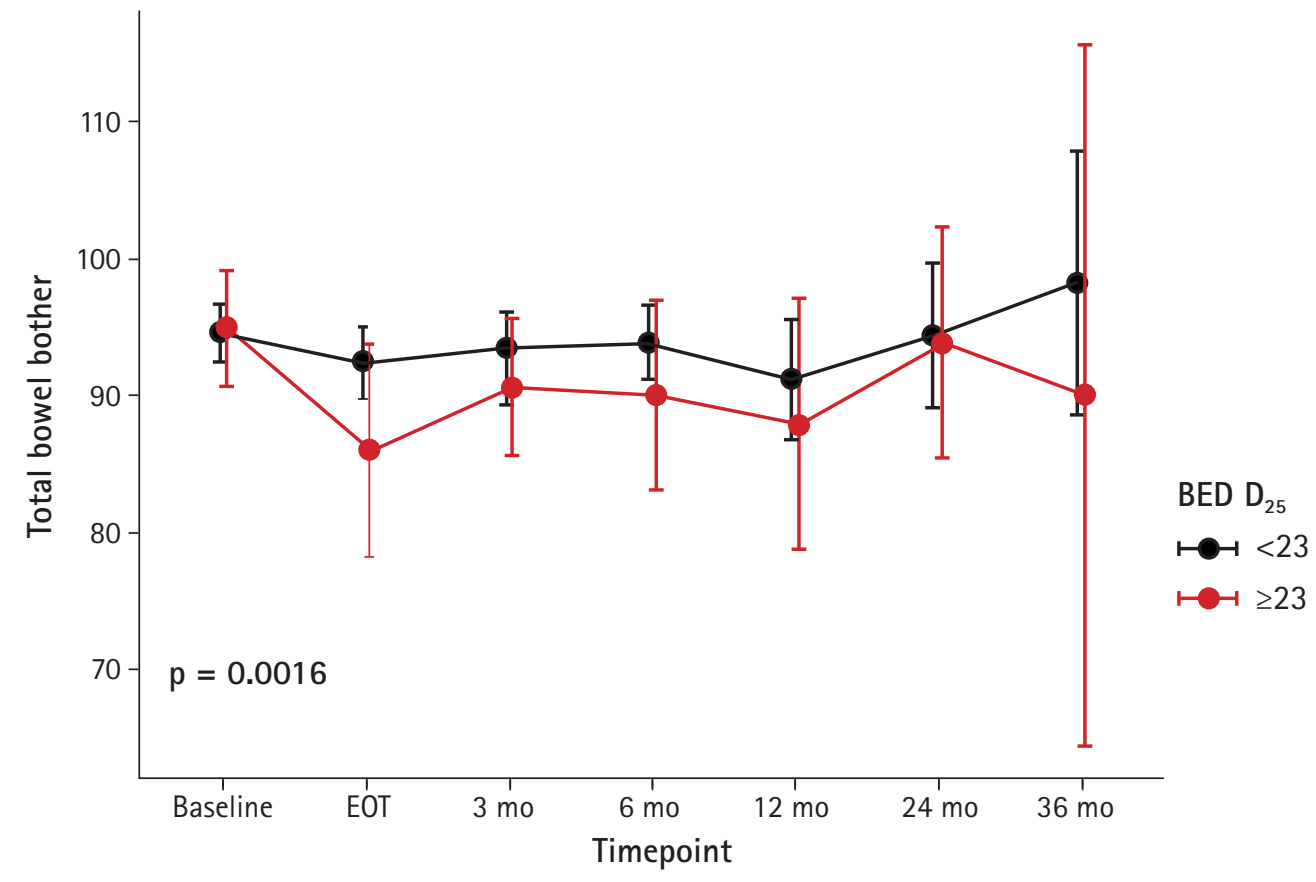

\begin{tabular}{|c|c|c|c|c|c|c|c|c|}
\hline $\mathfrak{0}^{2}$ & $\geq 23-$ & 51 & 42 & 35 & 38 & 30 & 18 & 5 \\
\hline \multirow[t]{3}{*}{ 무 } & \multirow[t]{3}{*}{$<23-$} & 176 & 151 & 120 & 114 & 94 & 43 & 12 \\
\hline & & Baseline & EOT & $3 \mathrm{mo}$ & $6 \mathrm{mo}$ & $12 \mathrm{mo}$ & $24 \mathrm{mo}$ & $36 \mathrm{mo}$ \\
\hline & & & & & Timep & & & \\
\hline
\end{tabular}

Fig. 1. EPIC-Bowel bother subdomain scores and survey samplings over time. EPIC, Expanded Prostate Cancer Index Composite; BED, biologically effective dose; EOT, end-of-treatment. 
burden. However, the MID has not specifically been established for the subdomains. We hypothesize that if the MID for the overall domain is 4 points then a 4.06 difference within the bother subdomain alone is clinically significant.

Potentially, these results can have different applications as we continue modifying radiation dose fractionations for the treatment of prostate cancer. Through the application of BED, we can minimize the risk of patient-reported toxicity regardless of dose fractionation, and compare different plans treated to different dose fractionation schemes. Since the toxicity is related to the biological effect, different doses with a similar biological effectiveness theoretically should impact $\mathrm{QOL}$ similarly.

We found extreme hypofractionation to be associated with a statistically and clinically significant decline in overall EPIC-Bowel scores, as well as individual function and bother scores, when compared to conventional fractionation ( $p<0.01$ ). Hypofractionation was not clinically significantly associated with a decrease in EPIC-Bowel scores-it was only found to be statistically significant for decrease in bowel function ( $p<0.01$ ) compared to conventional fractionation. These findings deserve further study into the effects of the separate fractionation schemes on QOL.

In our prior analysis, we observed a significant decline of the EPIC-Bowel domain associated with BED $V_{51}(G y)>14.3 \%(p=$ $0.01)$ and $\mathrm{EQD}_{2 \mathrm{~Gy}} \mathrm{~V}_{22}(\mathrm{~Gy})>16.4 \%$ ( $<$ < 0.003) [19]. We further developed this analysis by optimizing cut points for greatest effect on EPIC-Bowel scores, determining BED $D_{25}$ (Gy) $\geq 23 \%$ to be the most significant dosimetric predictor with the most clinically meaningful changes. Additionally, the present analysis evaluates function and bother subscales within the overall EPIC-Bowel domain, which serves to better capture the nature of $00 \mathrm{~L}$ decline within this patient set.

While the function subscale assessed topics such as bowel urgency, stool quality, and abdominal cramping, the bother subscale assessed how significantly these problems were perceived by patients. The differences in the scale suggest that patients have bother-related to radiation changes, but function was not affected. Thus, patients reported similar stool quality, but perception of the radiation effect on the change was larger than the perceived function. This can be interpreted in multiple ways: that patients can perceive differences in their stool quality for example, even though function is not affected, suggesting higher sensitivity to bother than a functional scale. Alternatively, the volume of the rectum that has developed radiation proctitis is relatively small. While the organ maintains function, but the small volume of rectum with proctitis can produce changes in bother in different subdomains. These findings may provide a more specific understanding of rectal toxicities associated with RT, guiding patient counseling.
Previous studies have supported the separate reporting of function and bother because treatment planning and symptom management may depend on a patients' perceived experience [20]. Perceived bother may vary between patients based on their individual concerns, as well as their preconceived expectations regardless of organ function, over the course of treatment $[21,22]$. In a study that prospectively analyzed EPIC-Bowel scores in response to photon radiotherapy $(n=228)$, on the subscales of function and bother, there were patterns of decline and resolution similar to our findings, with an association with urinary flare symptoms [23]. Another prospective study $(n=226)$ evaluated $\mathrm{OOL}$ and toxicity between passively scattered proton therapy and spot-scanning proton therapy, showing a significant decrement in bowel $00 \mathrm{~L}$ which persisted through follow-up [15]. Our study is unique because it validates the use of proton radiotherapy across various dose fractionation schemes. Through DVH analysis and statistical modeling that accounted for variations in treatment, patient characteristics, and demographics, this study demonstrates that BED, as a DVH tool, can be used to predict $\mathrm{QOL}$ across dose fractionations.

One limitation of the present study is the difficulty in administration of a 50-item questionnaire. Although the EPIC-50 was used in order to retain granularity in the data collection, this poses certain challenges related to survey fatigue in patients [24], observed by drop-rates in Fig. 1. Newer versions of the questionnaire, such as the EPIC-26 and EPIC-CP $[25,26]$, have been developed to remedy this. Another limitation is that our data was collected from patients exclusive to a single, high-volume academic institution. This could limit generalizability of the results in smaller practices. Furthermore, patients were treated according to physician preference, allowing for more variation in the analysis which may limit the power of certain subgroup analysis. We expect these findings to additionally be applicable to patients receiving photon RT, however different cut points may be necessary.

In summary, this study demonstrates that BED can be used as an independent predictor for bowel $\mathrm{QOL}$ across conventional fractionation, hypofractionation and extreme hypofractionation schemes. We specifically determined BED $D_{25}(G y) \geq 23 \%$ to be a statistically and clinically significant parameter for predicting decline in perceived bowel bother QOL. Therefore, BED can be a useful measure in prospective clinical trials to minimize symptom burden for patients undergoing PBT for prostate cancer, even when utilizing different dose fractionations.

\section{Conflict of Interest}

No potential conflict of interest relevant to this article was reported. 


\section{References}

1. Siegel RL, Miller KD, Jemal A. Cancer statistics, 2020. CA Cancer J Clin 2020;70:7-30.

2. Katz AJ, Kang J. Quality of life and toxicity after SBRT for organ-confined prostate cancer, a 7-year study. Front Oncol 2014; 4:301.

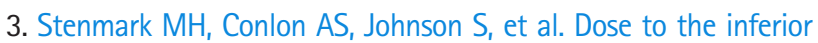
rectum is strongly associated with patient reported bowel quality of life after radiation therapy for prostate cancer. Radiother Oncol 2014;110:291-7.

4. Elias E, Helou J, Zhang L, et al. Dosimetric and patient correlates of quality of life after prostate stereotactic ablative radiotherapy. Radiother Oncol 2014;112:83-8.

5. Gomez $\mathrm{CL}$, Xu X, Qi XS, et al. Dosimetric parameters predict short-term quality-of-life outcomes for patients receiving stereotactic body radiation therapy for prostate cancer. Pract Radiat Oncol 2015;5:257-62.

6. Newhauser WD, Zhang R. The physics of proton therapy. Phys Med Biol 2015;60:R155-209.

7. Vargas C, Fryer A, Mahajan C, et al. Dose-volume comparison of proton therapy and intensity-modulated radiotherapy for prostate cancer. Int J Radiat Oncol Biol Phys 2008;70:744-51.

8. Sheets NC, Goldin GH, Meyer AM, et al. Intensity-modulated radiation therapy, proton therapy, or conformal radiation therapy and morbidity and disease control in localized prostate cancer. JAMA 2012;307:1611-20.

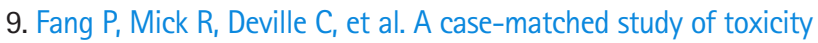
outcomes after proton therapy and intensity-modulated radiation therapy for prostate cancer. Cancer 2015;121:1118-27.

10. Santos PM, Barsky AR, Hwang WT, et al. Comparative toxicity outcomes of proton-beam therapy versus intensity-modulated radiotherapy for prostate cancer in the postoperative setting. Cancer 2019;125:4278-93.

11. Dutz A, Agolli L, Baumann M, et al. Early and late side effects, dosimetric parameters and quality of life after proton beam therapy and IMRT for prostate cancer: a matched-pair analysis. Acta Oncol 2019;58:916-25.

12. Wei JT, Dunn RL, Litwin MS, Sandler HM, Sanda MG. Development and validation of the expanded prostate cancer index composite (EPIC) for comprehensive assessment of health-related quality of life in men with prostate cancer. Urology 2000;56: 899-905.

13. Litwin MS, Hays RD, Fink A, Ganz PA, Leake B, Brook RH. The UCLA Prostate Cancer Index: development, reliability, and validity of a health-related quality of life measure. Med Care 1998; 36:1002-12.
14. Vargas CE, Hartsell WF, Dunn M, et al. Image-guided hypofractionated proton beam therapy for low-risk prostate cancer: analysis of quality of life and toxicity, PCG GU 002. Rep Pract Oncol Radiother 2016;21:207-12.

15. Pugh TJ, Munsell MF, Choi $S$, et al. Quality of life and toxicity from passively scattered and spot-scanning proton beam therapy for localized prostate cancer. Int J Radiat Oncol Biol Phys 2013;87:946-53.

16. van Leeuwen $C M$, Oei $A L$, Crezee J, et al. The alfa and beta of tumours: a review of parameters of the linear-quadratic model, derived from clinical radiotherapy studies. Radiat Oncol 2018;13:96.

17. Skolarus TA, Dunn RL, Sanda MG, et al. Minimally important difference for the Expanded Prostate Cancer Index Composite Short Form. Urology 2015;85:101-5.

18. Umbehr MH, Bachmann LM, Poyet $\mathrm{C}$, et al. The German version of the Expanded Prostate Cancer Index Composite (EPIC): translation, validation and minimal important difference estimation. Health Qual Life Outcomes 2018;16:36.

19. Bhangoo RS, DeWees TA, Petersen $M$, et al. Rectum dose-volume histogram (DVH) parameters and patient-reported EPIC-bowel domain for patients undergoing radiotherapy (RT) for prostate cancer (PC): a single-center prospective registry experience. Int J Radiat Oncol Biol Phys 2020;108:e852-3.

20. Reeve BB, Potosky AL, Willis GB. Should function and bother be measured and reported separately for prostate cancer quality-of-life domains? Urology 2006;68:599-603.

21. Aning JJ, Wassersug RJ, Goldenberg SL. Patient preference and the impact of decision-making aids on prostate cancer treatment choices and post-intervention regret. Curr Oncol 2012;19(Suppl 3):S37-44.

22. Gore JL, Gollapudi K, Bergman J, Kwan L, Krupski TL, Litwin MS. Correlates of bother following treatment for clinically localized prostate cancer. J Urol 2010;184:1309-15.

23. Bhattasali O, Chen LN, Woo J, et al. Patient-reported outcomes following stereotactic body radiation therapy for clinically localized prostate cancer. Radiat Oncol 2014;9:52.

24. Eysenbach G. The law of attrition. J Med Internet Res 2005;7:e11.

25. Szymanski KM, Wei JT, Dunn RL, Sanda MG. Development and validation of an abbreviated version of the expanded prostate cancer index composite instrument for measuring health-related quality of life among prostate cancer survivors. Urology 2010; 76:1245-50.

26. Chang P, Szymanski KM, Dunn RL, et al. Expanded prostate cancer index composite for clinical practice: development and validation of a practical health related quality of life instrument for use in the routine clinical care of patients with prostate cancer. J Urol 2011;186:865-72. 